\title{
Capacity Loss for Multilayer Codebook Precoding in MIMO Systems
}

\author{
Jawad Mirza*, Pawel A. Dmochowski*, Peter J. Smith ${ }^{\dagger}$, Mansoor Shafi ${ }^{\ddagger}$, \\ * School of Engineering and Computer Science, Victoria University of Wellington, New Zealand \\ $\dagger$ Department of Electrical and Computer Engineering, University of Canterbury, New Zealand \\ $\ddagger$ Telecom New Zealand, New Zealand \\ Email:\{jawad.mirza,pawel.dmochowski\}@ecs.vuw.ac.nz, mansoor.shafi@telecom.co.nz, p.smith@elec.canterbury.ac.nz
}

\begin{abstract}
In limited feedback multiple-input multiple-output (MIMO) systems it is important that the codebooks used provide a good approximation to the channel. Any mismatch between the codebook entries and the actual channel creates a non zero chordal distance (or subspace angle) between the codebook element and the corresponding columns of the right singular matrix of the channel. As a result, the system exhibits a capacity loss. We evaluate and quantify the amount of this capacity loss with long term evolution (LTE) and Grassmannian codebooks in the case of Layer 1 (L1) and Layer 2 (L2) transmission using linear receivers in three different channel models. We evaluate the distribution of the minimum distance for LTE and Grassmannian codebooks via simulations. We also derive capacity loss approximations for zero forcing (ZF), minimum mean-squared error (MMSE) and singular value decomposition (SVD) receivers. We find that the capacity loss approximations for $\mathrm{L} 1$ transmission are accurate for all receivers and lead to an extremely simple relationship between capacity performance and minimum distance.
\end{abstract}

\section{INTRODUCTION}

Codebook beamforming is now part of the LTE and LTE advanced standards [1]. The LTE standard uses specific codebooks referred to hereafter as LTE codebooks. However, there are other families of codebooks (eg. Grassmaninan codebooks [2], [3] and Vector Quantization codebooks [4]) that may also be used for limited feedback systems. There is a lot of interest in the performance of limited feedback (or codebook) beamforming. A comprehensive overview of limited or finiterate feedback systems is provided in [3]. Here, it is claimed that the benefits of limited feedback are nearly identical to having perfect channel knowledge at the transmitter provided that codebooks are judiciously designed.

Limited feedback systems often result in a capacity loss. There are many studies that deal with estimating and limiting this capacity loss. In the beamforming setting the capacity loss can be large if no power optimization via waterfilling is realized. In [5], a codebook design is achieved that results in a gain equivalent to waterfilling. In [6], a codebook design for a multimode (referred to as multilayer transmission in this paper) precoding scheme is proposed that bounds the capacity loss and even though it is based on a suboptimal precoding scheme, it outperforms the algorithm proposed in [5]. In [7] the effects of limited feedback on the performance of MIMO systems over an i.i.d. Rayleigh flat fading channel are studied. The paper derives tight lower bounds on the capacity loss due to limited feedback for the MIMO systems employing a fixed number of equal power spatial streams (layers). A similar study is also done in [8]. The cost of feedback is discussed in [9] where the authors present bounds on the amount of feedback required under different antenna, signal-to-noise ratio (SNR), and bandwidth assumptions. A bound on the ergodic capacity loss for a limited feedback MIMO system is given in [10] when random codebooks, generated from the uniform distribution on the complex unit sphere, are used. The authors provide a closed-form expression for the ergodic capacity loss as a function of the number of feedback bits. Some useful capacity loss expressions are derived for MIMO systems in [11], that explain the impact of capacity loss on codebook design, selection criteria and receiver design.

The key idea of limited feedback is that the receiver has correct channel knowledge but instead of feeding back the entire channel information to the transmitter, it compares the leading right singular vectors of the channel with entries in a codebook and feeds back the index of the nearest codebook entry. Clearly, one would want to find the codebook entry that is closest to the channel in terms of a metric such as chordal distance [3]. However, with limited numbers of codebook entries, it is rarely possible to find codebook entries that have a zero or negligible distance from the corresponding channel values. A non zero distance manifests itself as a capacity loss arising mainly due to interstream interference.

While there are a number of papers that determine or bound the capacity loss, or determine the number of feedback bits required to minimize the capacity loss, bulk of these papers consider an i.i.d. flat fading Rayleigh channel. Yet in practical deployments there is always some correlation present. Therefore, it will be useful to examine the capacity loss for codebooks that are already standardized and used in practical settings. The main theme of this paper is to look at the statistics of the minimum distance, $\mathrm{d}_{\min }$, and relate this to the capacity loss. The contributions in this paper are as follows:

- We derive, via simulation, the distribution of the minimum distance for LTE and Grassmannian codebooks with Layer 1 (L1) and Layer 2 (L2) transmissions.

- We derive an approximate capacity loss (CL) for Layer 1 (L1) and Layer 2 (L2) transmission respectively in terms of minimum distance.

- We simulate three types of channels, the iid channel, 
a Kronecker channel and the standard M.2135 channel for the urban macro (Uma) environment. For each, we simulate the performance of three types of receivers; zero forcing (ZF), minimum mean-squared error (MMSE) and singular value decomposition (SVD). We simulate two types of codebooks; LTE and Grassmanian. The approximate capacity loss expressions show good agreement for the simulations of L1 transmission for all cases described here. The use of L1 transmission is typically the case for practical systems.

\section{System ModeL}

We consider a single user, limited feedback MIMO-OFDM system with $n_{t}$ transmit and $n_{r}$ receive antennas, where, for a single subcarrier, the frequency flat fading channel is denoted by $\mathbf{H}$. The singular value decomposition (SVD) of $\mathbf{H}$ is $\mathbf{H}=\mathbf{U D V}^{\dagger}$, where $\mathbf{U} \in \mathbb{C}^{n_{r} \times n_{r}}$ and $\mathbf{V} \in \mathbb{C}^{n_{t} \times n_{t}}$ are unitary and $\mathbf{D}$ is a diagonal matrix containing the $n=$ $\min \left(n_{r}, n_{t}\right)$ singular values of $\mathbf{H}$. The transmitter encodes the data sequence, $\mathbf{b}$, by a precoding matrix, $\mathbf{P}$, selected from a codebook of quantized values of the first $L$ columns of $\mathbf{V}$, denoted by $\mathbf{V}_{\mathrm{c}}=\left[\mathbf{V}_{\mathrm{c}_{1}}, \mathbf{V}_{\mathrm{c}_{2}}, \ldots, \mathbf{V}_{\mathrm{c}_{M}}\right]$. Thus, for $L \leq n$ transmitted data streams, the codebook consists of $M$ $n_{t} \times L$ codewords used to precode the $L \times 1$ data vector $\mathbf{b}$. Denoting by $\mathbf{V}(k)$ and $\mathbf{V}_{\mathbf{c}_{i}}(k)$ the $k^{t h}$ column of $\mathbf{V}$ and $\mathbf{V}_{\mathrm{c}_{i}}$, respectively, the codeword selection is performed according to the minimum subspace angle ${ }^{1}$, which is given by

$$
\mathrm{d}_{\text {min }}=\min _{\mathbf{V}_{\mathrm{c}_{i}} \in \mathbf{V}_{\mathbf{c}}}\left\{L-\sum_{k=1}^{L}\left|\mathbf{V}^{\dagger}(k) \mathbf{V}_{\mathbf{c}_{i}}(k)\right|^{2}\right\},
$$

and thus, for the selected codeword $\mathbf{V}_{\mathrm{c}_{i}}$, the $k^{t h}$ component $^{2}$ of $d_{\min }$ is

$$
d_{\min _{k}}=1-\left|\mathbf{V}^{\dagger}(k) \mathbf{V}_{\mathrm{c}_{i}}(k)\right|^{2} .
$$

The index of the selected codeword is fed back to the transmitter in the form of a precoder matrix index (PMI) and thus $\mathbf{P}=\mathbf{V}_{\mathrm{c}_{(\mathrm{PMI})}}$. The resulting system equation is given by

$$
\mathbf{r}=\mathbf{H P b}+\mathbf{n},
$$

where $\mathbf{n}$ is a vector of iid complex Gaussian $\mathcal{C N}\left(0, \sigma^{2}\right)$ random variables. The total transmit power is $P_{T}=\mathbb{E}\left(\mathbf{b}^{\dagger} \mathbf{b}\right)$ so that for each stream $\mathbb{E}\left(\left|\mathbf{b}_{k}\right|^{2}\right)=P_{T} / L$ and we assume a zero mean i.i.d. structure for $\mathbf{b}$. The noise power for each stream is $\mathbb{E}\left(\left|\mathbf{n}_{k}\right|^{2}\right)=\sigma^{2}$ so the link SNR is $\rho=P_{T} / \sigma^{2}$. The received vector $\mathbf{r}$ is decoded by means of a linear combiner $\mathbf{W}$ to produce $\tilde{\mathbf{r}}=\mathbf{W}^{\dagger} \mathbf{r}$. We consider three types of receivers, namely the SVD receiver $\left(\mathbf{W}_{\mathrm{SVD}}=\mathbf{U}\right)$, as well as the zero forcing (ZF) and minimum-mean-squared error (MMSE) receivers, for which $\mathbf{W}$ is given by

$$
\mathbf{W}_{\mathrm{ZF}}=\mathbf{H P}\left(\mathbf{P}^{\dagger} \mathbf{H}^{\dagger} \mathbf{H} \mathbf{P}\right)^{-1}
$$

\footnotetext{
${ }^{1}$ The formal definition of chordal distance involves taking the square root of (1). The selection of codeword based on minimum chordal distance or minimum subspace angle is equivalent.

${ }^{2}$ We note that the minimization of $d_{\min }$ does not necessarily result in each component $d_{\min _{k}}$ being minimized.
}

and

$$
\mathbf{W}_{\mathrm{MMSE}}=\mathbf{H} \mathbf{P}\left(\frac{L}{\rho} \mathbf{I}+\mathbf{P}^{\dagger} \mathbf{H}^{\dagger} \mathbf{H} \mathbf{P}\right)^{-1},
$$

respectively.

\section{CAPACITY LoSS ANALYSIS}

We now derive the signal-to-interference-and-noise ratio (SINR) and capacity loss results as a function of the minimum codebook distances, $d_{\min _{k}}$, for the receivers described in Section II. We will focus on the most common scenarios, namely one and two-stream transmission, referred to as Layer 1 (L1) and Layer 2 (L2).

\section{A. ZF/MMSE Receivers}

The SINR of the $k^{t h}$ stream in the ZF receiver is given by [12], [13]

$$
\gamma_{k}=\frac{\rho}{L\left[\left(\mathbf{P}^{\dagger} \mathbf{H}^{\dagger} \mathbf{H P}\right)^{-1}\right]_{k k}},
$$

which can be written as

$$
\gamma_{k}=\frac{\rho}{L\left[\left(\mathbf{P}^{\dagger} \mathbf{V} \mathbf{\Lambda} \mathbf{V}^{\dagger} \mathbf{P}\right)^{-1}\right]_{k k}},
$$

where $\boldsymbol{\Lambda}$ is a diagonal matrix containing the eigenvalues $\lambda_{k}$ of $\mathbf{H}^{\dagger} \mathbf{H}$.

1) L2 transmission: Taking the inverse in (7), we can, after some manipulation, express the SINR of the ZF receiver with L2 transmission as

$$
\gamma_{k}=\frac{\rho}{L}\left[\sum_{i=1}^{n} \lambda_{i}\left|\alpha_{k, i}\right|^{2}-\frac{\left|\sum_{i=1}^{n} \lambda_{i} \alpha_{k, i} \alpha_{m, i}^{\dagger}\right|^{2}}{\sum_{i=1}^{n} \lambda_{i}\left|\alpha_{m, i}\right|^{2}}\right]_{\substack{m=1,2 \\ m \neq k}},
$$

where we have defined $\alpha_{k, i}=\mathbf{P}(k)^{\dagger} \mathbf{V}(i)$. We can further approximate (8) by disregarding the second term, giving

$$
\gamma_{k} \approx \frac{\rho}{L}\left[\sum_{i=1}^{n} \lambda_{i}\left|\alpha_{k, i}\right|^{2}\right] .
$$

In the high SNR regime, the performance of the ZF and MMSE receivers are similar, therefore, (8) and (9) are also valid for the MMSE receiver with L2 transmission. Isolating the $k^{\text {th }}$ stream in the summation in (9) and using (1) we can express (9) in terms of $d_{\min _{k}}$ as

$$
\gamma_{k_{\text {(ZFMMSE) }}} \approx \frac{\rho}{L}\left[\lambda_{k}\left(1-d_{\text {min }_{k}}\right)+\sum_{i=1, i \neq k}^{n} \lambda_{i}\left|\alpha_{k, i}\right|^{2}\right] .
$$

The capacity for the linear receivers is given in terms of their respective stream SINR values as

$$
C=\sum_{k=1}^{L} \log _{2}\left(1+\gamma_{k}\right)
$$

We define capacity loss (CL) for all receivers as the difference between the capacity with perfect feedback and the capacity with codebook feedback. Hence, 


$$
\mathrm{CL}=\sum_{k=1}^{L}\left[\log _{2}\left(1+\rho \lambda_{k} / L\right)-\log _{2}\left(1+\gamma_{k}\right)\right] .
$$

The total capacity loss for the ZF and MMSE receivers at high SNR with L2 transmission can be approximated using (8) as

$$
\begin{aligned}
& \mathrm{CL}_{\text {(ZF/MMSE) }} \approx \sum_{k=1}^{2}-\log _{2}\left[1-d_{\min _{k}}+\frac{1}{\lambda_{k}} \times\right. \\
& \left.\left(\sum_{i=1, i \neq k}^{n} \lambda_{i}\left|\alpha_{k, i}\right|^{2}-\frac{\left|\sum_{i=1}^{n} \lambda_{i} \alpha_{k, i} \alpha_{m, i}^{\dagger}\right|^{2}}{\sum_{i=1}^{n} \lambda_{i}\left|\alpha_{m, i}\right|^{2}}\right)\right] \substack{m=1,2 \\
m \neq k}_{\substack{m \neq k \\
m}} .
\end{aligned}
$$

A simplified expression can be obtained by substituting (10) in (12), giving

$$
\begin{array}{r}
\mathrm{CL}_{\text {(ZF/MMSE) }} \approx \sum_{k=1}^{2}-\log _{2}\left[1-d_{\min _{k}}+\right. \\
\left.\frac{1}{\lambda_{k}} \sum_{i=1, i \neq k}^{n} \lambda_{i}\left|\alpha_{k, i}\right|^{2}\right] .
\end{array}
$$

2) L1 transmission: In the case of L1 transmission, the second term in (8) is zero and the SINR of the $1^{\text {st }}$ stream is the same as (9)

$$
\gamma_{1} \approx \frac{\rho}{L}\left[\sum_{i=1}^{n} \lambda_{i}\left|\alpha_{1, i}\right|^{2}\right]
$$

which can be expressed in terms of minimum distance as

$$
\gamma_{1_{\text {(ZFMMSE) }}} \approx \rho\left(1-d_{\text {min }_{1}}\right) \lambda_{1},
$$

where we neglected the second term in (10). The capacity loss for ZF and MMSE receivers with L1 transmission is obtained by substituting (16) in (12), which, for high SNR, gives

$$
\mathrm{CL}_{\text {(ZF/MMSE) }} \approx-\log _{2}\left(1-d_{\text {min }_{1}}\right) \text {. }
$$

\section{B. SVD Receiver}

The SVD receiver in a MIMO channel is more susceptible to interstream interference as compared to the $\mathrm{ZF}$ and MMSE receivers.

1) L2 transmission: For L2 transmission, using (3) we have that the received symbol on the $k^{t h}$ stream is

$$
\tilde{\mathbf{r}}_{k}=\sqrt{\lambda_{k}} \mathbf{V}^{\dagger}(k) \mathbf{P b}+\tilde{\mathbf{n}}_{k},
$$

where $\mathbf{P}=[\mathbf{P}(1) \mathbf{P}(2)], \mathbf{b}=\left[\begin{array}{ll}b_{1} & b_{2}\end{array}\right]^{T}$ and $\tilde{\mathbf{n}}_{k}$ is the $k^{\text {th }}$ element of $\tilde{\mathbf{n}}=\mathbf{U}^{\dagger} \mathbf{n}$. From (18), we have that the desired signal and interstream interference for the $1^{\text {st }}$ stream are $\sqrt{\lambda_{k}} \mathbf{V}^{\dagger}(1) \mathbf{P}(1) b_{1}$ and $\sqrt{\lambda_{k}} \mathbf{V}^{\dagger}(1) \mathbf{P}(2) b_{2}$ respectively, and similarly the SINR of the SVD receiver for the $k^{t h}$ stream is

$$
\gamma_{k_{\text {(SVD) }}}=\left[\frac{\lambda_{k} \mathbb{E}\left(\left|\mathbf{b}_{k}\right|^{2}\right)\left|\mathbf{V}(k)^{\dagger} \mathbf{P}(k)\right|^{2}}{\lambda_{k} \mathbb{E}\left(\left|\mathbf{b}_{m}\right|^{2}\right)\left|\mathbf{P}(m)^{\dagger} \mathbf{V}(k)\right|^{2}+\sigma^{2}}\right]_{\substack{m=1,2 \\ m \neq k}} .
$$

We can further simplify (19) by using $\mathbb{E}\left(\left|\mathbf{b}_{k}\right|^{2}\right)=\mathbb{E}\left(\left|\mathbf{b}_{m}\right|^{2}\right)=$ $P_{T} / L$ and $\rho=P_{T} / \sigma^{2}$. Now we can express (19) in terms of minimum distance using (2) as

$$
\gamma_{k \text { (SVD) }}=\left[\frac{1-d_{\min _{k}}}{\left|\alpha_{m, k}\right|^{2}+L / \rho \lambda_{k}}\right]_{\substack{m=1,2 \\ m \neq k}} .
$$

Note that $\gamma_{k_{\text {(SVD) }}} \rightarrow\left(1-d_{\text {min }_{k}}\right) /\left|\alpha_{m, k}\right|^{2}$ as $\rho \rightarrow \infty$, so there is an upper limit on the SINR due to interference. This results in a capacity loss which grows with SNR as seen in (21) and Sec. IV. The total capacity loss approximated at high SNR for an SVD receiver with L2 transmission is obtained by using (20) in (12) to give

$$
\mathrm{CL}_{(\mathrm{SVD})} \approx \sum_{k=1}^{2} \log _{2}\left[\frac{\rho\left|\alpha_{m, k}\right|^{2} \lambda_{k} / L}{\left|\alpha_{m, k}\right|^{2}+1-d_{\min _{k}}}\right]_{\substack{m=1,2 \\ m \neq k}} .
$$

2) L1 transmission: In the case of L1 transmission, there is no interstream interference and $\left|\alpha_{m, k}\right|^{2}=0$ in (20). Thus, we have

$$
\gamma_{1_{\text {(SVD) }}} \approx \rho\left(1-d_{\min _{1}}\right) \lambda_{1} .
$$

In contrast to (20), $\gamma_{1_{\text {(SVD) }}}$ is unbounded as $\rho \rightarrow \infty$. Hence, the capacity loss converges to a limit as seen in (23) and Sec. IV. We can express the capacity loss for the SVD receiver in L1 transmission as

$$
\mathrm{CL}_{(\mathrm{SVD})} \approx-\log _{2}\left(1-d_{\min _{1}}\right) .
$$

Note that the capacity loss expression for the SVD receiver is identical to that of the ZF and MMSE receivers (17) for L1 transmission.

\section{Simulation Results}

In this section, we provide system simulation results for i.i.d., Kronecker and M.2135 channel models. The MIMO system simulated has four antennas each at transmitter and receiver using either L1 or L2 transmission. The Kronecker channel is highly correlated with the exponential correlation coefficient $\mathrm{r}=0.9$ at both ends. The M.2135 channel is designed for IMT Advanced systems by the ITU-R to simulate wireless mobile channels [14]. In this paper, the M.2135 channel is based on the WINNER II MATLAB channel simulations [15]. The total power gain $\mathbb{E}\left(\operatorname{Tr}\left[\mathbf{H H}^{\dagger}\right]\right)=n_{r} n_{t}$ of the channel matrix is the same for all three channels. The codebooks used in the simulation are 4 bit LTE and Grassmannian codebooks and the feedback link is assumed lossless with zero delay. We calculate the capacity of the MIMO system by taking the mean of the capacity for all the OFDM tones and then averaging it over all the time samples. The parameters for the M.2135 channel model are given in Table I. 
TABLE I

M.2135 SimUlation PARAMETERS

\begin{tabular}{|l|c|}
\hline Scenario & UMa \\
\hline Frequency Band & $2.1 \mathrm{GHz}$ \\
\hline Bandwidth & $20 \mathrm{MHz}$ \\
\hline Propagation & $\mathrm{NLoS}$ \\
\hline Antenna configuration & $4 \times 4$ slant pol \\
\hline Antenna spacing & $4 \lambda @ \mathrm{Tx}, 0.5 \lambda @ \mathrm{Rx}$ \\
\hline MS speed & $5 \mathrm{~km} / \mathrm{h}$ \\
\hline Sampling density & 4 samples per $\lambda$ \\
\hline
\end{tabular}

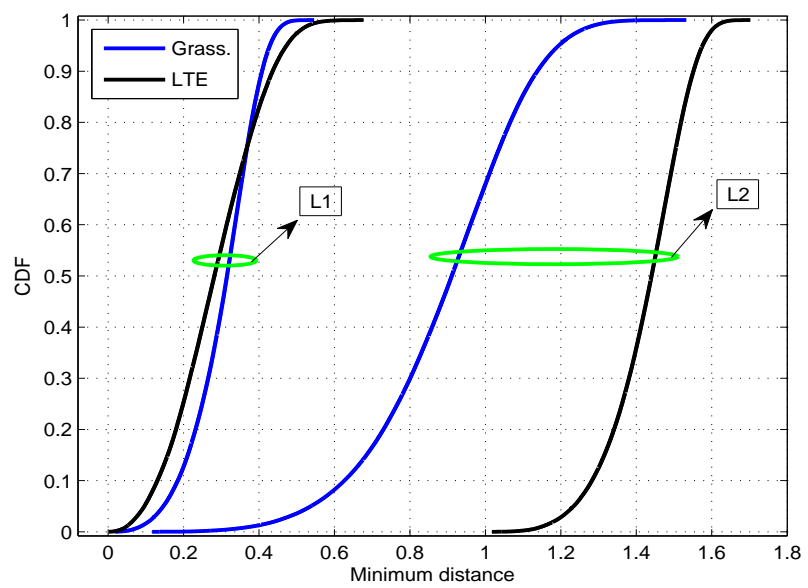

Fig. 1. Minimum distance CDF with LTE and Grassmannian (4 bit) codebooks for L1 and L2 transmission in the M.2135 channel.

In Fig. 1, we see the cumulative distribution function (CDF) of the minimum distance, $\mathrm{d}_{\min }$, for both $\mathrm{L} 1$ and L2 transmissions with LTE and Grassmannian codebooks in the M.2135 channel. The minimum distance is smaller in L1 transmission with the LTE codebook compared to the Grassmannian codebook. However, for L2 transmission, the Grassmannian codebook yields a much smaller minimum distance than the LTE codebook. The average minimum distance for L1 and L2 transmission with all the three channels and both the codebooks is given in Table II.

In Fig. 2, ergodic capacity (EC) results for all three receivers with the LTE codebook are presented for different channels. The correlated channels improves the EC in the L1 transmission. This is due to the reason that the dominant eigen mode becomes more dominant in correlated channels. Therefore, the M.2135 and Kronecker channels are providing better capacity results than the i.i.d. channel in L1 transmission.

TABLE II

Average Minimum Distance

\begin{tabular}{|c|c|c|c|c|c|c|}
\hline \multirow{2}{*}{} & \multicolumn{2}{|c|}{ M.2135 } & \multicolumn{2}{c|}{ i.i.d. } & \multicolumn{2}{c|}{ Kronecker } \\
\cline { 2 - 7 } & LTE & Grass. & LTE & Grass. & LTE & Grass \\
\hline $\mathrm{d}_{\min }$ L1 & 0.28 & 0.30 & 0.34 & 0.30 & 0.18 & 0.30 \\
\hline $\mathrm{d}_{\min }$ L2 & 1.43 & 0.90 & 1.45 & 0.90 & 1.44 & 0.90 \\
\hline
\end{tabular}

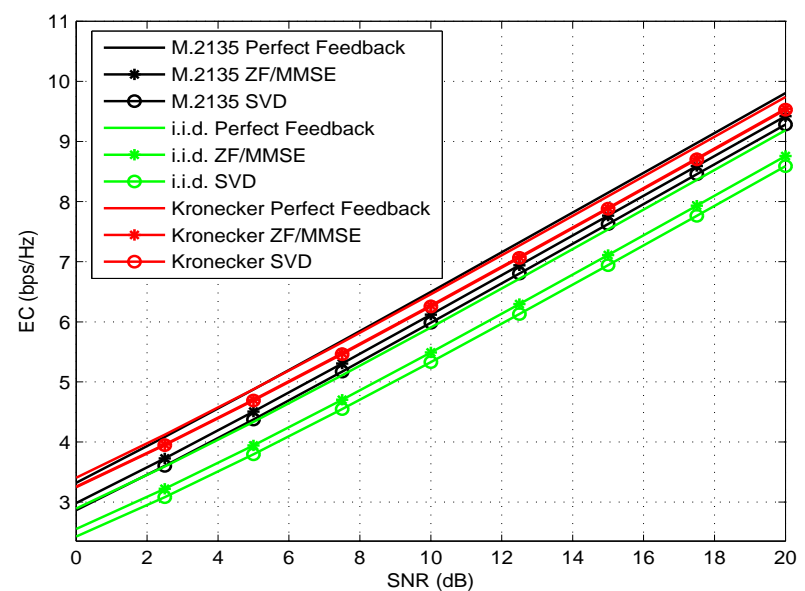

Fig. 2. Ergodic Capacity for L1 transmission with LTE (4 bit) codebook.

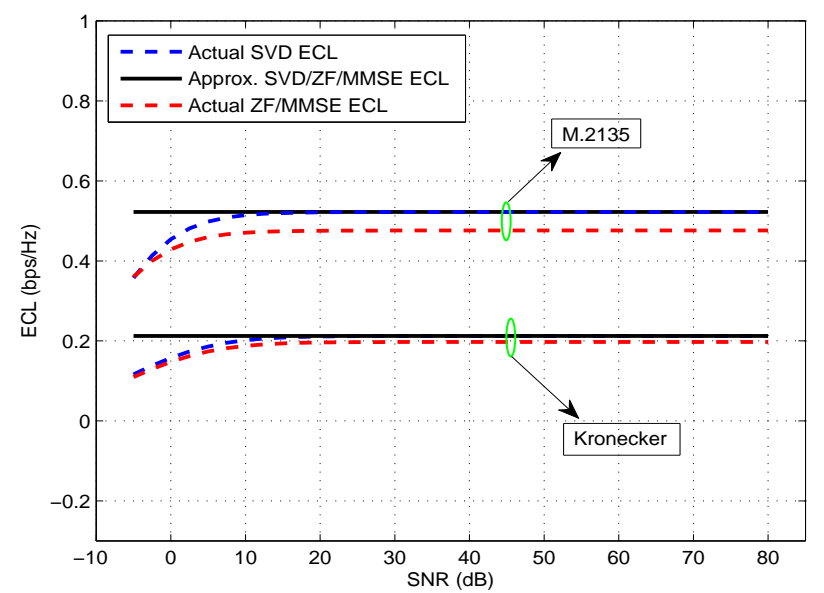

Fig. 3. Ergodic Capacity Loss for SVD, ZF snd MMSE receivers with L1 transmission and LTE (4 bit) codebook in M.2135 and Kronecker channels.

Figure 3 shows the ergodic capacity loss (ECL) for L1 transmission with the LTE codebook for M.2135 and Kronecker channels. The approximated capacity loss is calculated using (23). It is seen that (23) approximates the SVD receiver ECL accurately at high SNR, but a small offset between the actual and approximated ECL for the ZF and MMSE receivers is the result of ignoring the contribution towards signal power from the other unused layers.

In Fig. 4, we provide the EC results for all three receivers with L2 transmission in the M.2135 channel. The EC with the Grassmannian codebook is larger than the EC with the LTE codebook for both ZF and MMSE receivers. But at high SNR, EC for the SVD receiver is the same for both codebooks. Fig. 5 shows the EC results for all three receivers with L2 transmission in the Kronecker channel. The Kronecker channel is highly correlated $(\mathrm{r}=0.9)$ and it is seen that at low SNR there is a significant difference between the $\mathrm{EC}$ of the $\mathrm{ZF}$ and MMSE receivers. In the high SNR regime, the EC of the ZF 


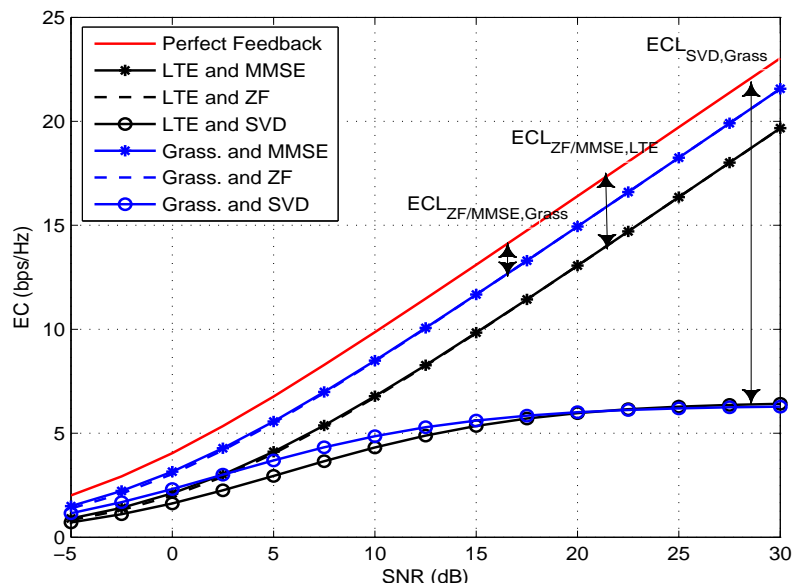

Fig. 4. Ergodic Capacity for L2 transmission with LTE and Grassmannian (4 Bits) codebooks in the M.2135 channel.

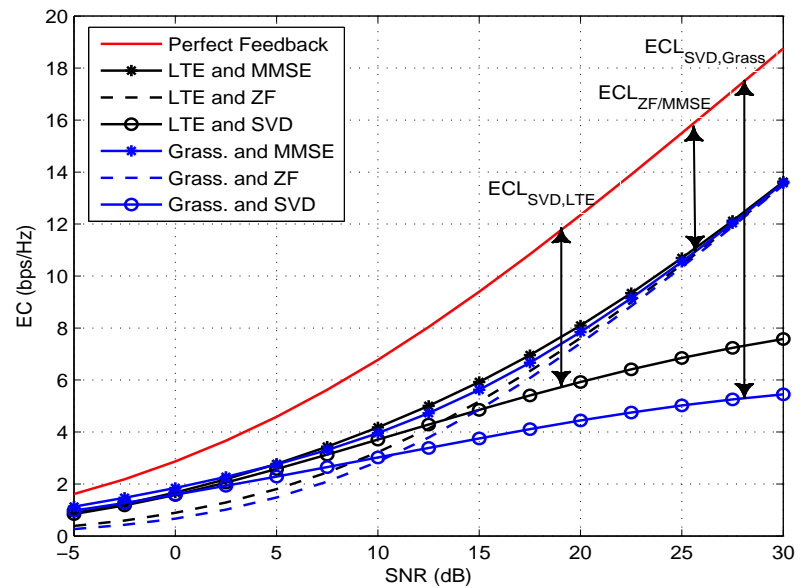

Fig. 5. Ergodic Capacity for L2 transmission with LTE and Grassmannian (4 Bits) codebooks in the Kronecker channel.

and MMSE receivers is equal for both codebooks. The LTE codebook gives better capacity results than the Grassmannian codebook for the SVD receiver at high SNR in the Kronecker channel.

Figure 6 shows the ECL for the ZF and MMSE receivers in the case of L2 transmission. As we are not including the effect of interference from other unused layers in (14), the approximate ECL values have a $13 \%$ and $28 \%$ error with respect to actual ECL values for LTE and Grassmannian codebooks respectively. On the other hand, (13) approximates the capacity loss perfectly at high SNR for both the codebooks.

In Fig. 7, the ECL for the SVD receiver is shown with L2 transmission in the M.2135 channel. The approximated capacity loss is calculated using (21). In the case of L2 transmission, the use of (21) in the high SNR regime provides the best ECL approximation for the SVD receivers. The ECL for the SVD receiver increases with the SNR in L2

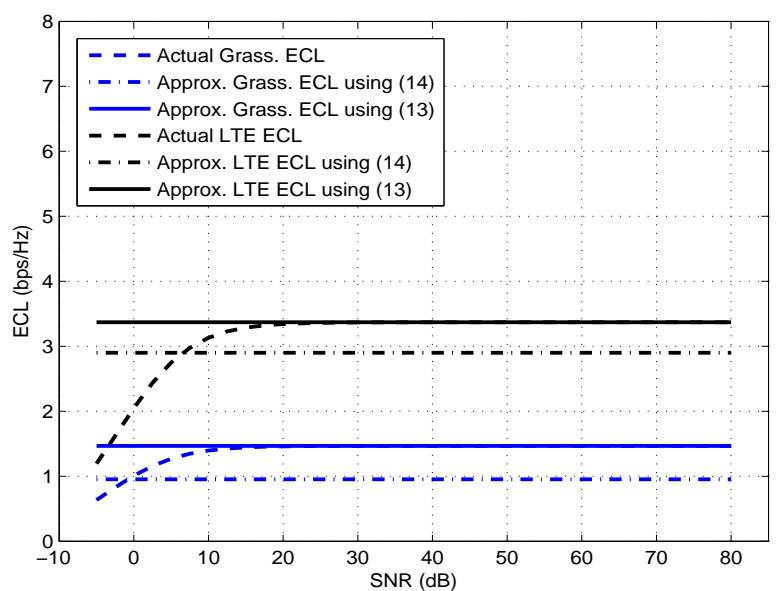

Fig. 6. Ergodic Capacity Loss for ZF snd MMSE receivers with L2 transmission and LTE (4 Bits) codebooks in the M.2135 channel.

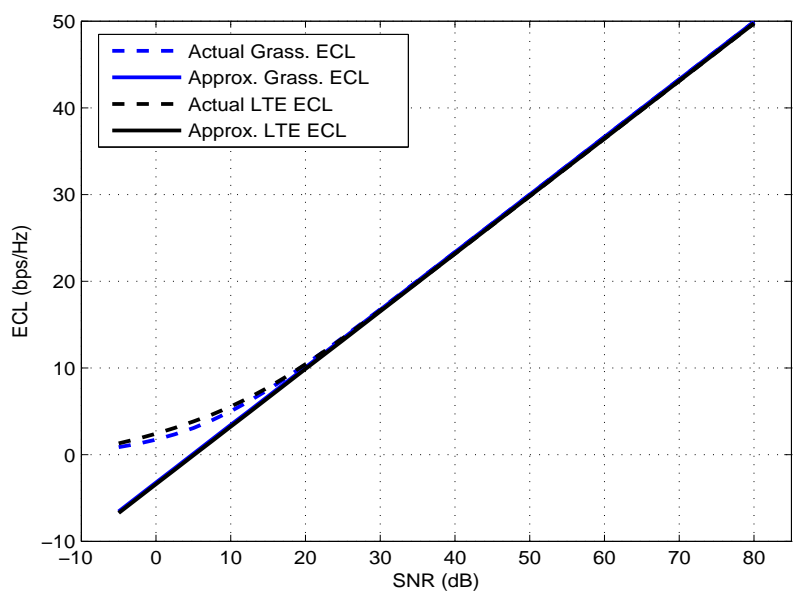

Fig. 7. Ergodic Capacity Loss for SVD L2 transmission with LTE and Grassmannian (4 Bits) codebooks in the M.2135 channel.

transmission, as shown in Fig. 4 and Fig. 5, whereas, ECL for the ZF and MMSE receivers remains constant in the high SNR regime.

\section{CONClusion}

In this paper, we have derived an approximate expression for the capacity loss for a MIMO system that uses multilayer transmission based on codebook beamforming. The capacity loss approximation is expressed in terms of the minimum distance between the codebook entry selected and corresponding channel values and is valid for high SNR. The accuracy of this approximation is compared for $\mathrm{L} 1$ and $\mathrm{L} 2$ transmission via simulation of three kinds of channels; iid channel, Kronecker channel; and an industry standard M.2135 channel and for three types of receivers (ZF, MMSE and SVD). The approximation is in agreement for both layers for the SVD receiver but slightly under-estimates the loss for the other receiver types, especially for L2 transmission. In this regard, it could be used 
as lower bound. However, in practice, one is likely to only use L1 transmission for which the loss approximation is good. The approximation therefore provides a useful measure to relate the capacity loss to codebook design. Further work in this area will focus on determining the statistics of the minimum distance and thus determining the statistics of the loss.

\section{REFERENCES}

[1] 3GPP TS 36.211, "Evolved universal terrestrial radio access (E-UTRA), physical channels and modulation," vol. V10.0.0, Jan. 2011.

[2] D. J. Love, R. W. Heath Jr., and T. Strohmer, "Grassmannian beamforming for multiple-input multiple-output wireless systems," IEEE Trans. on Inform. Theory, vol. 49, no. 10, pp. 2735 - 2747, Oct. 2003.

[3] D. J. Love, R. W. Heath Jr., V. K. N. Lau, D. Gesbert, B. D. Rao, and M. Andrews, "An overview of limited feedback in wireless communication systems," IEEE J. on Sel. Areas in Com., vol. 26, no. 8, pp. $1341-1365$, Oct. 2008.

[4] J. Roh and B. Rao, "Transmit beamforming in multiple-antenna systems with finite rate feedback: a VQ-based approach," IEEE Trans. on Inf Theory, vol. 52, no. 3, pp. 1101 - 1112, March 2006.

[5] V. Lau, Y. Liu, and T.-A. Chen, "On the design of MIMO blockfading channels with feedback-link capacity constraint," IEEE Trans. on Commun., vol. 52, no. 1, pp. 62 - 70, Jan. 2004.

[6] X. Song and H.-N. Lee, "Multimode precoding for MIMO systems: Performance bounds and limited feedback codebook design," IEEE Trans. on Signal Processing, vol. 56, no. 10, pp. 5296 - 5301, Oct. 2008.

[7] J. Zheng and B. Rao, "Capacity analysis of MIMO systems using limited feedback transmit precoding schemes," IEEE Trans. on Signal Processing, vol. 56, no. 7, pp. 2886 - 2901, July 2008.

[8] J. Roh and B. Rao, "Design and analysis of MIMO spatial multiplexing systems with quantized feedback," IEEE Trans. on Signal Processing, vol. 54, no. 8, pp. 2874 - 2886, Aug. 2006.

[9] D. Love, "Duplex distortion models for limited feedback MIMO communication," IEEE Trans. on Signal Processing, vol. 54, no. 2, pp. 766 - 774, Feb. 2006.

[10] A. Dabbagh and D. Love, "Feedback rate-capacity loss tradeoff for limited feedback MIMO systems," IEEE Trans. on Inf. Theory, vol. 52, no. 5, pp. 2190 - 2202, May 2006.

[11] M. Zhang, M. Shafi, P. Smith, and P. Dmochowski, "Precoding performance with codebook feedback in a MIMO-OFDM system," in Proc. IEEE Int. Conf. Commun., vol. 52, June 2011, pp. $1-6$.

[12] A. Paulraj, R. Nabar, and D. Gore, Introduction to Space-time Wireless Communications. Cambridge Univ Pr, 2003.

[13] D. Love and R. Heath Jr, "Limited feedback precoding for spatial multiplexing systems using linear receivers," in Proc. IEEE Military Commun. Conf., vol. 1, Oct. 2003, pp. 627 - 632.

[14] ITU, "Guidelines for evaluation of radio interface technologies for IMTAdvanced," Report ITU-R M.2135, 2008

[15] L. Hentilä, P. Kyösti, M. Käske, M. Narandzic, and M. Alatossava, "Matlab implementation of the WINNER Phase II channel model ver1.1 [online]," IST-WINNERII Project, Dec, 2007. 